\title{
Innovations in geriatric medicine: inception and dissemination of a national trainee-led research collaborative
}

\author{
Author: Geriatric Medicine Research Collaborative
}

\author{
Aims \\ Primary aim. \\ > To set up the first national trainee-led geriatric medicine \\ research collaborative. \\ Secondary aims. \\ > To enable conduct of audit and research projects that intend to \\ have direct impact on patient care. \\ > To enable clinical trainees in geriatric medicine to develop \\ valuable research skills.
}

\section{Methods}

We set up a national trainee-led Geriatric Medicine Research Collaborative (GeMRC) with trainee representatives from across the UK, to improve access to and understanding of research. Our collaborative has been trainee-led from inception through to project management and conduct.

We created national and regional websites to disseminate project information. We utilised a combination of email communication, social networking, word-of-mouth, and dissemination through the British Geriatrics Society (BGS). Project ideas were generated by trainees at an initial meeting and communicated to regional representatives. This approach encouraged engagement of trainees, including those with minimal research experience. Our first meeting led to generation of ideas, which were subsequently presented and discussed at the NIHR Clinical Research Network. This trainee-led 'grass-roots' approach has encouraged early participation.

One of the pivotal concepts of our research collaborative is that all members and researchers are considered equal. Our approach to contribution is flexible allowing trainees to be involved in the process of study design, obtaining ethical approval, public involvement, participant recruitment, analysis of results and dissemination. Trainees may choose to be involved in all or part of these processes. However, in order to formalise communication, we have developed a formal structure of network, regional, and local leads to support individual participation.

\section{Results}

We have been successful in obtaining representatives for 13 out of 15 training regions within the UK within 3 months of creation. We have also successfully begun collaboration with other specialty networks, including orthopaedics, and general surgery. We have successfully completed two national audit projects, and we are in the process of refining the protocols for two randomised controlled trials, four abstracts have been submitted to the BGS national conference, one article has been submitted to a peer-review journal and more than 100 trainees have been involved in this initiative.

\section{Conclusion}

The GeMRC offers an innovative approach to research conduct. GeMRC facilitates the opportunity for trainees with limited research experience to participate in research conduct, and to complete research with an early impact upon patient care.

Trainee-led research collaboratives, driven by online networking and social media are an innovative approach to conducting research. The use of social media and online networking allows rapid dissemination of project ideas and involvement of multiple regions early in the development of a collaborative. This facilitates collection of much larger datasets and enhances the scientific validity of project outcomes.

A particularly innovative approach has been to create a separate website specifically for the purpose of communicating our research ideas, project design and results to non-medically trained individuals. We hope this 'public engagement section' will engage service users, carers and the general public to become involved in generating research ideas which can then evolve through the GeMRC network to produce patient-initiated projects.

\section{Conflict of interest statement}

There are no conflicts of interest. 